ANDRZEJ ŚWIERNIAK (Gliwice)

\title{
COMPARISON OF SIX MODELS OF ANTIANGIOGENIC THERAPY
}

Abstract. Six models of antiangiogenic therapy are compared and analyzed from control-theoretic point of view. All of them consist of a model of tumor growth bounded by the capacity of a vascular network developed by the tumor in the process of angiogenesis and different models of dynamics of this network, and are based on the idea proposed by Hahnfeldt et al. Moreover, we analyse optimal control problems resulting from their use in treatment protocol design.

1. Introduction. Angiogenesis is a complex process which leads to the formation of new vessels from existing ones and it is stimulated and controlled by molecular factors called activators (stimulators) and inhibitors (blockers) of angiogenesis. During progression of tumor these factors are released by tumor itself to develop its own vascular network which enables its growth and in the next stage determines the possibility of cancer metastasis. Since this network is necessary for tumor development, in the late sixties of the last century a new anticancer therapy was proposed, targeted not directly at the cancer cells but at the new born vascular network. This therapy is known as antiangiogenic therapy and the idea is to reduce the tumor volume by reducing its vasculature. It was first hypothesized by Folkman $(1971,1972)$ more than thirty years ago. Folkman's main suggestions are as follows:

a) primary solid tumors go through a prolonged state of avascular growth (almost quiescent) in which the maximum attainable size is 1-2 $\mathrm{mm}$ in diameter, and the necessary oxygen and nutrients are supplied by passive diffusion,

2000 Mathematics Subject Classification: 93C10, 92C50, 49N90, 93C95, 37N25.

Key words and phrases: biomathematical modelling, optimal control, nonlinear systems, dynamic systems, cancer. 
b) these microscopic tumors can switch on angiogenesis by recruiting surrounding mature host blood vessels to start sprouting new blood vessel capillaries which grow and infiltrate the tumor mass, thus setting the potential for metastatic spread,

c) the angiogenic switch is triggered by elaboration by tumor cells of a growth factor (TAF),

d) blocking tumor angiogenesis factors or simply destroying newly formed immature blood vessels may be used to affect tumor growth.

The most important obstacle against successful chemotherapy is drug resistance acquired by cancer cells while the normal tissues retain sensitivity to the drugs.

This negative feature of chemotherapy may be used as an advantage in the antiangiogenic therapy which is directed towards a special part of normal tissues and only indirectly destroys tumor cells and that is why it has been called by Kerbel (1997) a therapy resistant to drug resistance. Therapy directed against tumor vasculature does not exploit tumor cell sensitivity, relying instead on tumor suppression consequent to inhibition of associated vasculature. For more than ten years Folkman's ideas were not followed by experimental or clinical investigations but now tumor angiogenesis belongs to the most inspiring areas of cancer research. Kerbel (2000) presents ten significant reasons for the explosive growth in tumor angiogenesis research and development of antiangiogenic drugs (we follow the order of items proposed by Kerbel):

1) The discovery of basic fibroblast growth factor as the first pro-angiogenic molecule (Folkman and Klagsburn, 1987).

2) The discovery of vascular endothelial growth factor and its receptor tyrosine kinases on activated endothelial cells (Klagsburn and Soker, 1993).

3) The discovery of angiopoietins and their tyrosine kinase receptors (Davis and Yancopoulos, 1999).

4) The discovery of endogenous inhibitors of angiogenesis (Folkman, 1995).

5) The discovery of additional molecular markers in newly formed blood vessels (Bischoff, 1995).

6) The development of quantitative assays for angiogenesis (Folkman and Haudebschild, 1980).

7) Recognition of the prognostic significance of tumor angiogenesis (Weidner, 1995).

8) Lack of acquired resistance to directly acting antiangiogenic drugs (Kerbel, 2000).

9) The discovery of the impact of angiogenesis on liquid hematologic malignancies (D'Amato et al., 1994). 
10) The discovery of the accidental antiangiogenic effects of various conventional or new anticancer drugs (Denekamp, 1993).

The complexity of the process of vascularization as well as the way in which inhibitors, stimulators and antiangiogenic drugs act results in complex models (see e.g. Mantzaris et al., 2004) applicable for simulation of the process but less useful in synthesis or even analysis of therapy protocols. The exception is a class of models proposed by Hahnfeldt et al. (1999) who suggested that the tumor growth with incorporated vascularization mechanism can be described by a Gompertz type or logistic type equation with variable carrying capacity which defines the dynamics of the vascular network. Roughly speaking, the main idea of this class of models is to incorporate the spatial aspects of the diffusion of factors that stimulate and inhibit angiogenesis into a non-spatial two-compartmental model for cancer cells and vascular endothelial cells. The models considered here belong to this class.

\section{Models of cancer growth including vascularization and an-} tiangiogenic therapy. The simplest model of population kinetics for cancer tissues is given by Malthusian growth which assumes exponential relationship between the size of the population and time. The dynamics is described by the equation

$$
\dot{N}=a N, \quad N(0)=N_{0},
$$

resulting in the following form of the solution:

$$
N=N_{0} e^{a t}, \quad a=\ln 2 / P D T,
$$

with $N$ denoting the size of the population and $a$ Malthusian parameter defined by the inverse of the potential doubling time $(P D T)$. The unlimited growth in this model can be avoided if we introduce a varying coefficient $a(t)$ as in the Gompertz model:

$$
\begin{aligned}
\dot{N} & =a(t) N, \quad N(0) & =N_{0}, \\
\dot{a} & =-\beta a, \quad a(0) & =\alpha .
\end{aligned}
$$

The solution has the following form:

$$
N=N_{0} e^{\alpha / \beta\left(1-e^{-\beta t}\right)} .
$$

The growth is bounded by

$$
N_{\infty}=N_{0} e^{\alpha / \beta},
$$

which is called the carrying capacity in population dynamics. The same solution is obtained when we use the non-linear Gompertz equation in the form

$$
\dot{N} / N=-\beta \ln N / N_{\infty} \approx 1 / P D T .
$$


Hahnfeldt et al. (1999) proposed to treat the carrying capacity which constrains the tumor growth as a varying tumor volume sustainable by the vessels and roughly proportional to the vessel volume:

$$
\begin{aligned}
N_{\infty} & =K, \\
\dot{N} / N & =-\beta \ln N / K .
\end{aligned}
$$

Although the equations (6) and (7) appear similar, the carrying capacity is not constant in (7) but varies with changes of the volume of the vessels.

Similar behavior may be obtained if the Gompertz type growth is substituted by a logistic one (also called Pearl-Verhulst growth). Then we have

$$
\dot{N} / N=\beta(1-N / K) \text {. }
$$

The dynamics of the growth of the volume $K$ represented by its $P D T$ depends on the stimulators of angiogenesis $(S F)$, inhibitory factors secreted by tumor cells $(I F)$ and natural mortality of the endothelial cells $(M F)$ :

$$
P D T_{k}=f(M F, S F, I F) \text {. }
$$

In Hahnfeldt et al. (1999) it has been assumed that the inverse of PDT is the sum of these three factors, i.e.

$$
1 / P D T_{k}=M F+S F+I F .
$$

The spontaneous loss of functional vasculature represented by $M F$ (e.g. through natural mortality of the endothelial cells) is supposed to be negative constant, the stimulatory capacity of the tumor upon inducible vasculature represented by $S F$ (e.g. through angiogenic factors like the vascular endothelial factor) is found to grow at rate $K^{b} N^{c}$ slower than the endogenous inhibition of previously generated vasculature represented by $I F$ (e.g. through endothelial cell death or disaggregation) where

$$
b+c \sim 2 / 3 .
$$

This results from the assertion that tumor-driven inhibitors from all sites act more systematically whereas tumor-derived stimulators act more locally to the individual secreting tumor site. On the other hand, analyzing a diffusionconsumption equation for the concentration of stimulator or inhibitor inside and outside the tumor, Hahnfeldt et al. concluded that the inhibitor will influence target endothelial cells in the tumor in a way that grows ultimately as the area of the active surface between the tumor and the vascular network, which in turn is proportional to the square of the tumor diameter. This leads to the conclusion that $I F$ is proportional to the tumor volume to the power $2 / 3$ since the volume is proportional to the cube of the diameter. The expression for $K$ suggested in Hahnfeldt et al. (1999) has therefore the following form:

$$
\dot{K} / K=\gamma N / K-\left(\lambda N^{2 / 3}+\mu\right),
$$


$\gamma, \lambda, \mu$ being constant parameters representing the effect of stimulation, inhibition and natural mortality, respectively. The modification of this model proposed in d'Onofrio and Gandolfi (1999) which also satisfies Hahnfeldt's suggestions given by (11) assumes that the effect of $S F$ and $M F$ on the inverse of $P D T_{K}$ is constant while $I F$ is proportional to the active surface of the area of tumor being in contact with the vascular network (as in the original Hahnfeldt model):

$$
\dot{K} / K=\gamma-\left(\lambda N^{2 / 3}+\mu\right) .
$$

Combinations of tumor growth models $(7),(8)$ with vascular network models $(12),(13)$ result in the following four nonlinear models of tumor angiogenesis:

- A (original Hahnfeldt model)

$$
\dot{N} / N=-\beta \ln N / K, \quad \dot{K} / K=\gamma N / K-\left(\lambda N^{2 / 3}+\mu\right) .
$$

- B (Hahnfeldt model with logistic growth)

$$
\dot{N} / N=\beta(1-N / K), \quad \dot{K} / K=\gamma N / K-\left(\lambda N^{2 / 3}+\mu\right) .
$$

- C (d'Onofrio-Gandolfi model with Gompertz type growth)

$$
\dot{N} / N=-\beta \ln N / K, \quad \dot{K} / K=\gamma-\left(\lambda N^{2 / 3}+\mu\right) .
$$

- D (d'Onofrio-Gandolfi model with logistic growth)

$$
\dot{N} / N=\beta(1-N / K), \quad \dot{K} / K=\gamma-\left(\lambda N^{2 / 3}+\mu\right) .
$$

Yet another simplified model was proposed by Ergun et al. (2003):

$$
\dot{K} / K=\gamma K^{-1 / 3}-\lambda K^{1 / 3} .
$$

In this case the growth of the vascular network is independent of the tumor size. Nevertheless to have a complete model of the tumor growth in the vascular stage we should add one of the two previously proposed models of growth (Gompertz or logistic type) and thus we are led to two additional models:

- E (Ergun model with Gompertz type growth)

$$
\dot{N} / N=-\beta \ln N / K, \quad \dot{K} / K=\gamma K^{-1 / 3}-\lambda K^{1 / 3},
$$

and

- F (Ergun model with logistic growth)

$$
\dot{N} / N=\beta(1-N / K), \quad \dot{K} / K=\gamma K^{-1 / 3}-\lambda K^{1 / 3} .
$$

An interesting finding is that the systems $\mathrm{A}-\mathrm{D}$ have the same nontrivial equilibrium point $\left(N^{*}, K^{*}\right)$ :

$$
\dot{N} / N=\dot{K} / K=0 \Rightarrow N^{*}=K^{*}=((\gamma-\mu) / \lambda)^{3 / 2} .
$$


The models are strongly nonlinear but by a logarithmic change of variables and some scaling transformations we are able to simplify them and find their asymptoptic properties using standard Lyapunov type analysis of stability (local and global) following the line of reasoning presented by d'Onofrio and Gandolfi (1999).

More precisely, by the transformation

$$
\begin{aligned}
x & =\ln N / N^{*}, & & y=\ln K / K^{*}, \\
x^{*} & =y^{*}=0, & & \tau=\beta t, \\
\vartheta & =(\gamma-\mu) / \beta, & & \\
x^{\prime} & =d x / d \tau, & y^{\prime} & =d y / d \tau,
\end{aligned}
$$

we are led:

- for model $\mathrm{C}$, to the following quasi-linear system:

$$
x^{\prime}=y-x, \quad y^{\prime}=-\vartheta\left(e^{2 x / 3}-1\right),
$$

or to

$$
z=y-x, \quad x^{\prime}=z, \quad z^{\prime}=-z-\vartheta\left(e^{2 x / 3}-1\right),
$$

and for models A, B, D to slightly more complicated systems, namely:

- for model B,

$$
z=1-e^{x-y}, \quad x^{\prime}=z, \quad z^{\prime}=(z-1)\left(z(1+\gamma / \beta)+\vartheta\left(e^{2 x / 3}-1\right)\right),
$$

- for model A,

$$
z=y-x, \quad x^{\prime}=z, \quad z^{\prime}=-z-\frac{\gamma}{\beta}\left(1-e^{-z}\right)-\vartheta\left(e^{2 x / 3}-1\right),
$$

- for model D,

$$
z=1-e^{x-y}, \quad x^{\prime}=z, \quad z^{\prime}=(z-1)\left(z+\vartheta\left(e^{2 x / 3}-1\right)\right) .
$$

For all models using the first method of Lyapunov (linearization method) we find that the system is locally asymptotically stable in the neighbourhood of the nontrivial equilibrium point $\left(x^{*}=0, y^{*}=0\right)$ or equivalently $\left(N^{*}, K^{*}\right)$. Global stability can be proved by the second method of Lyapunov (see e.g. La Salle and Lefschetz, 1961) using the Lyapunov function of Lurie type representing the total energy of the system. For models A and C such a function has the following form (see d'Onofrio and Gandolfi, 1999):

$$
V(x, z)=0.5 z^{2}+\vartheta \int_{0}^{x}\left(e^{2 \xi / 3}-1\right) d \xi .
$$

Since $\left(e^{2 x / 3}-1\right) x>0$ for all $x \neq 0$, the Lyapunov function is positive definite and radial bounded.

Moreover, for model $\mathrm{C}, V^{\prime}=-z^{2} \leq 0$, which in turn represents dissipation of the system. Since in the subspace $z=0$ the equilibrium point $(0,0)$ 
is the only invariant set, the La Salle invariance theorem (La Salle and Lefschetz, 1961) implies that the equilibrium point is globally asymptotically stable.

The same Lyapunov function used for model A yields

$$
V^{\prime}=-z^{2}-\frac{\gamma}{\beta} z\left(1-e^{-z}\right) \leq 0,
$$

and the same argument as before leads to the conclusion about global asymptotic stability of the equilibrium point.

For models B and D similar results may be obtained using the Lyapunov function

$$
V=-z-\ln (1-z)+\vartheta \int_{0}^{x}\left(e^{2 \xi / 3}-1\right) d \xi
$$

which once more represents the total energy of the system. The definition of this function could be obtained from the transformed form of the differential equations for the variable $z$ in these models. More precisely, we can write the last equation of (19) in the form

$$
z^{\prime} /(z-1)=\left(z(1+\gamma / \beta)+\vartheta\left(e^{2 x / 3}-1\right)\right),
$$

and the last equation of (21) in the form

$$
z^{\prime} /(z-1)=\left(z+\vartheta\left(e^{2 x / 3}-1\right)\right) .
$$

Since always $z<1$, the Lyapunov function is always positive definite and for both cases $V^{\prime}=-d z^{2} \leq 0$, with $d=1+\gamma / \beta$ or $d=1$ respectively. Following the line of reasoning used for models $\mathrm{A}$ and $\mathrm{C}$ we are led to the same conclusions. In the case of Ergun's model, analysis of stability becomes trivial because of the independence of the vascular network growth from the tumor growth.

Application of antiangiogenic therapy can be incorporated into the model by a factor increasing multiplicatively the mortal loss rate of the vessels. For example in the case of the model (13) this leads to the following equation:

$$
\dot{K} / K=\gamma-\left(\lambda N^{2 / 3}+\mu+\eta u(t)\right),
$$

where $u(t)$ denotes the dose of the agent scaled to its effect on vascular network and $\eta$ is a constant parameter. For the constant dose $U$, the equilibrium points take the form

$$
N^{*}=K^{*}=((\gamma-\mu-\eta U) / \lambda)^{3 / 2},
$$

which according to the conditions of stability given by d'Onofrio and Gandolfi (1999) leads to the following conclusion:

$$
U=(\gamma-\mu) / \eta \Rightarrow K^{*} \rightarrow 0 .
$$


In other words, the vascular network and in turn the tumor can be eradicated. This conclusion is crucial for the philosophy of the entire analysis. It is enough to ensure that the population of endothelial cells responsible for angiogenesis behaves in the required way because the size of the tumor population in some sense tracks the same transients. D'Onofrio and Gandolfi proved that the same effect might be reached for periodic therapy with mean value satisfying condition (26) or greater. Nevertheless this condition is generally only necessary and not sufficient since for model A eradication of the tumor depends on the shape of pulses in the periodic protocol. For the other models this condition is both necessary and sufficient. For example, in the case of model $\mathrm{C}$ one of the following conditions should be satisfied:

1) If $U>(\gamma-\mu) / \eta$ then $K(t) \leq K(0) e^{(\gamma-\mu-\eta U) t} e^{-F(t)} \rightarrow 0$ for $t \rightarrow \infty$;

2) If $U=(\gamma-\mu) / \eta$ then $\dot{K}=\left(-\eta f-\lambda N^{2 / 3}\right) K$, which implies $K(t) \leq$ $K(0) e^{-\lambda \int_{0}^{t} N^{2 / 3}(\tau) d \tau} e^{-F(t)} \rightarrow 0$ for $t \rightarrow \infty$,

for periodic therapy with average value and variability defined as

$$
U=\frac{1}{T} \int_{0}^{T} u(t) d t, \quad f(t)=u(t)-U, \quad F(t)=\eta \int_{0}^{t} f(\tau) d \tau .
$$

Ergun's model in the case when therapy is included has the form

$$
\dot{K} / K=\gamma K^{-1 / 3}-\eta u-\lambda K^{1 / 3},
$$

or after the logarithmic transformation of variable $K$ :

$$
\dot{y}=\gamma e^{-y / 3}-\lambda e^{y / 3}-\eta u .
$$

Although during simulation all six models lead to a similar evolution while uncontrolled, their behaviour in the presence of control, modelling different therapeutic protocols, may differ significantly. Moreover, clinical interpretation of the results is also sensitive to the choice of the model.

3. Optimization of therapy in a finite horizon. Constant or periodic therapies which ensure tumor eradication discussed previously have an important drawback. They should be applied for a long therapy horizon. Shortage in antiangiogenic drugs, their costs, and side effects cause that the parameters of treatment protocols and the cumulated dose of the drugs should be bounded. A reasonable solution is to formulate an optimal control problem for the system given by the proposed model and the control objective which adequately represents the primary goal of the therapy. In Ergun et al. (2003) and more rigorously in Ledzewicz and Schättler (2005) the optimal control problem for the Ergun model with Gompertz type tumor growth (our model E) and a free terminal time is solved. The authors found that an optimal strategy consists of bang-bang (i.e. with the control switching between maximal and minimal values) and singular intervals (with 
intermediate values of the control variable, see e.g. Krener, 1977). Świerniak et al. (2006a) have proposed, for model C, to optimize the protocol in a fixed finite time of therapy with the primary goal of finding the control that maximizes TCP (tumor cure probability). This approach leads to the following equivalent form of an optimal control problem:

$$
\begin{aligned}
& J=N\left(T_{k}\right), \quad \int_{0}^{T_{k}} u(t) d t \leq \Xi, \\
& 0 \leq u(t) \leq U_{m},
\end{aligned}
$$

with known constraining constant parameters $U_{m}, \Xi$. Due to isoperimetric form of the optimization problem it could be transformed into a problem with an integral part of the performance index instead of an integral constraint on the control. Moreover, we may use the transformed variables $x$ and $y$ (or $x$ and $z$ ) to formulate the modified performance criterion in the form

$$
\begin{aligned}
& I=g x\left(T_{f}\right)+h y\left(T_{f}\right)+r \int_{0}^{T_{f}} u(\tau) d \tau, \\
& 0 \leq u \leq 1, \quad T_{f}=T_{k} \beta
\end{aligned}
$$

where the state variables are defined by the equations depending on the model chosen from among the six models mentioned. Although $J$ and $I$ are not the same indices and the respective optimization problems are not completely equivalent, the qualitative behaviour of their solutions should be given by the same optimal strategies because of the monotonic transformations of variables. For model $\mathrm{C}$ we have

$$
\begin{aligned}
x^{\prime} & =y-x, \\
y^{\prime} & =-\vartheta\left(e^{2 x / 3}-1\right)+\sigma u, \\
\sigma & =-\eta / \beta .
\end{aligned}
$$

The weight coefficients $h, g, r$ may change in broad ranges depending on the type of therapy used and the strength of the integral constraint. The additional term related to the volume of vascular network may be regarded as yet another constraint imposed on the possible dynamics of the system. On the other hand, by the choice of the weighting coefficients we obtain a new possibility of analysis of the mutual dependence between the tumor growth and the volume of the vascular network. Thus it is reasonable to provide an extensive analysis of their effect on the solution of the optimal control problem. Necessary conditions of optimality can be found using the Pontryagin maximum principle (Pontryagin et al., 1964) for Hamiltonian 
and co-state variables $p, q$ defined as

$$
\begin{array}{cc}
H=r u+\sigma q u+p(y-x)-q \vartheta\left(e^{2 x / 3}-1\right), \\
p^{\prime}=p+(2 / 3) q \vartheta e^{2 x / 3}, & p\left(T_{f}\right)=g, \\
q^{\prime}=-p, & q\left(T_{f}\right)=h .
\end{array}
$$

This leads to the following switching function and bang-bang control law:

$$
q=-r / \sigma>0, \quad u=\left\{\begin{array}{l}
1 \\
0
\end{array} \Leftarrow \min H .\right.
$$

Rewriting the co-state equation in the form of a scalar second order ODE we have

$$
q\left(T_{f}\right)=h .
$$

An important finding is that singular arcs are not feasible since there are no finite intervals of constant solutions to the co-state equation. This leads to the conclusion that intermediate doses of the drug are not optimal and that the optimal protocol contains only switches between maximal dose and no drug intervals. This allows one to find recurrently the solution of the TPBVP composed of the state and co-state equations with bang-bang control found from the switching condition by using for example the shooting algorithm.

Ledzewicz and Schättler (2006) solved the optimal control problem for the Hahnfeldt original model (model A) in a similar way to the Ergun model (model E) and once more proved that in the optimal strategy some parts are singular. We are able to prove that a reasonable reformulation of the optimization problem for of from the six models enables avoidance of singular arcs and leads to pure bang-bang solutions. The only exception is model A where the optimal solution may contain a singular control as a middle part of the control strategy.

In the case of the Hahnfeldt model with logistic type growth of the tumor (model B) we may define

$$
z=\ln K N^{\theta}, \quad \theta=\gamma / \beta, \quad x=\ln N, \quad \varepsilon=\lambda / \beta .
$$

This leads to the following state equations:

$$
x^{\prime}=1-e^{(\theta+1) x-z}, \quad z^{\prime}=\vartheta-\varepsilon e^{2 x / 3}+\sigma u .
$$

For simplicity we assume $h=0$ in the performance index. Thus the Hamiltonian has the form

$$
H=r u+\sigma q u+p\left(1-e^{(\theta+1) x-z}\right)+q\left(\vartheta-\varepsilon e^{2 x / 3}\right)
$$

and co-state variables are given by

$$
\begin{array}{ll}
p^{\prime}=p(\theta+1) e^{(\theta+1) x-z}+(2 / 3) q \varepsilon e^{2 x / 3}, & p\left(T_{f}\right)=g, \\
q^{\prime}=-p e^{(\theta+1) x-z}, & q\left(T_{f}\right)=0 .
\end{array}
$$


Thus the necessary conditions of optimality have the form identical to (34). Once more the singular arcs are not feasible since there are no finite intervals of constant solutions to the co-state equation. For the d'Onofrio-Gandolfi model with the logistic type tumor growth (model D) the analysis is similar. We use the state equations

$$
x^{\prime}=1-e^{x-y}, \quad y^{\prime}=\vartheta\left(e^{2 x / 3}-1\right)+\sigma u,
$$

and the performance index (30). This leads to the following Hamiltonian and co-state equations:

$$
\begin{aligned}
& H=r u+\sigma q u+p\left(1-e^{x-y}\right)+q \vartheta\left(e^{2 x / 3}-1\right), \\
& p^{\prime}=p e^{x-y}+(2 / 3) q \vartheta e^{2 x / 3}, \quad p\left(T_{f}\right)=g, \\
& q^{\prime}=-p e^{x-y}, \quad q\left(T_{f}\right)=h .
\end{aligned}
$$

Once more the optimal control strategy has the form (34) and singular arcs are not present.

For the Ergun model the solution is even simpler if the optimization problem is reformulated in the following way. We choose $g=0$ returning to the primary goal of the antiangiogenic therapy, i.e. eradication of the vascular network created in the process of tumor angiogenesis. Since the equation defining $y$ is independent of $x$ we are led to a first order optimization problem which has no singular solutions. The solution in this case is independent of the tumor growth and therefore it is the same for models E and F.

The problem is defined by the state equation

$$
\dot{y}=\gamma e^{-y / 3}-\lambda e^{y / 3}-\eta u,
$$

and the Hamiltonian and the co-state variable are given by

$$
\begin{aligned}
H & =p\left(\gamma e^{-y / 3}-\lambda e^{y / 3}\right)+(r-\eta p) u, \\
\dot{p} & =\frac{1}{3} p\left(\gamma e^{-y / 3}+\lambda e^{y / 3}\right), \quad p\left(T_{k}\right)=h .
\end{aligned}
$$

Thid leads to the following form of the bang-bang candidate for optimality:

$$
p=r / \eta>0, \quad u=\left\{\begin{array}{l}
1 \\
0
\end{array} \Leftarrow \min H,\right.
$$

and singular controls cannot be optimal for the same reasons as in the previously analyzed problems. The only model in which singular arcs as parts of optimal trajectory could not be eliminated is the standard Hahnfeldt model with Gompertz type tumor growth (model A), as proved rigorously by Ledzewicz and Schättler (2007). But from this point of view it is an exception rather than a rule. If we recall that this model has also different properties from the others in the case of periodic therapy (dependence of its asymptotic behaviour on the shape of pulses) we may try to find what is behind this specificity of this model. 
4. Discussion and concluding remarks. In this study we have compared different modifications of the Hahnfeldt model of vascular tumor growth and their application to rationales of antiangiogenic therapy. We consider advantages and drawbacks of six such models in the context of their possible application and difficulties of mathematical analysis. We also discuss results of some other authors and discuss possible approaches to optimization problems arising from therapy protocol design. All considerations are however based on the assumption that the complex phenomena leading to vessel collapse and regression could be described by such simplified models. It should be interesting to check how robust they are by testing them on the much more complex models recently published (e.g. Bartha and Rieger, 2006). Tumor angiogenesis belongs to the most inspiring areas of cancer research. Nevertheless still the most important constrain in efficient antiangiogenic therapy is the accessibility of antiangiogenic agents. This is why the rational anticancer therapy should contain a combination of antiangiogenic therapy with more standard modalities of anticancer treatment, for example radiotherapy. We discuss a model of such combined therapy in Świerniak (2007) for the d'Onofrio-Gandolfi model.

The effect of radiotherapy should be included in both compartments of the model because radiation destroys both cancer and normal tissues. The classical LQ model (e.g. Thames and Hendry, 1987) assumes that the damage to DNA, which is the principal target of the radiation, has two components: a linear one that is a consequence of a simultaneous break in both DNA strands caused by a single radiation particle, and a quadratic one that is the result of two separate but adjacent breaks in different strands caused by two different particles. In our model we omit this second term and introduce only linear (in dose) effect into both equations. This leads to the following model:

$$
\begin{aligned}
& \dot{N} / N=-\beta \ln N / K-\psi v, \\
& \dot{K} / K=\gamma-\left(\lambda N^{2 / 3}+\mu\right)-\eta u-\xi v,
\end{aligned}
$$

where $v(t)$ denotes the dose of radiotherapy scaled to its effect on tumor and normal tissues, and $\xi$ and $\psi$ are constant scaling parameters. Of course the additional radiotherapy supports the effect of antiangiogenic therapy. Moreover the effect of tumor eradication may be achieved in an easier and faster way although the theoretical results based on the theory of stability still have asymptotic form.

In Ergun et al. (2003) the optimal control problem for Ergun's model was presented for a free terminal time.

In our study we have proposed the same optimization scheme as discussed above for the antiangiogenic therapy. This leads to the following 
equivalent form of an optimal control problem:

$$
\begin{aligned}
& J=N\left(T_{k}\right), \quad \int_{0}^{T_{k}} u(t) d t \leq \Xi, \quad \int_{0}^{T_{k}} v(t) d t \leq \Phi, \\
& 0 \leq u(t) \leq U_{m}, \quad 0 \leq v(t) \leq V_{m}
\end{aligned}
$$

with known constraining constant parameters: $U_{m}, V_{m}, \Xi, \Phi$. The integral constraints imposed on the control variables, although similar in form, have a different meaning. For radiotherapy the constraint measures the feasible cumulated negative effect of the radiation while the one for antiangiogenic agent represents mostly the shortage in the availability of the agent and only in part the possible side effects of the drugs (not sufficiently recognized yet). Due to isoperimetric form of the problem it could be transformed into the problem with an integral part of the performance index instead of an integral constraint on the control:

$$
J=N\left(T_{k}\right)+r \int_{0}^{T_{k}} u(t) d t+s \int_{0}^{T_{k}} v(t) d t, \quad 0 \leq u \leq U_{m}, \quad 0 \leq v \leq V_{m} .
$$

This problem in turn can be approximated by a quasilinear problem in logarithmic variables as discussed in Section 3. Once more singular arcs for antiangiogenic therapy are not feasible since there are no finite intervals of constant solutions to the adjoint equation (the solution is the same as the one discussed in Section 3). On the other hand, singular arcs for radiotherapy do not satisfy the Legendre-Clebsch condition.

More precisely, for the state equations in the form

$$
x^{\prime}=y-x-v v, \quad y^{\prime}=\vartheta\left(1-e^{2 x / 3}\right)+\sigma u+\zeta v,
$$

and performance index

$$
I=g x\left(T_{f}\right)+h y\left(T_{f}\right)+r \int_{0}^{T_{f}} u(\tau) d \tau+s \int_{0}^{T_{f}} v(\tau) d \tau
$$

(where $\zeta=-\xi / \beta$ and $v=\psi / \beta$ ), we have the following co-state equations:

$$
\begin{array}{ll}
p^{\prime}=p+\frac{2}{3} q \vartheta e^{2 x / 3}, & p\left(T_{f}\right)=g, \\
q^{\prime}=-p, & q\left(T_{f}\right)=h,
\end{array}
$$

switching functions $f_{s}$ and bang-bang candidates for optimality:

$$
\begin{array}{ll}
q=-r / \sigma, & u=\left\{\begin{array}{l}
1 \\
0
\end{array} \text { min } H,\right. \\
p=s / v+q \zeta / v, & v=\left\{\begin{array}{l}
1 \\
0
\end{array} \Leftarrow \min H .\right.
\end{array}
$$


Thus (51) is the same as (34), leading to the same conclusions about optimal antiangiogenic strategy while for (52) we can use the Legendre-Clebsch necessary conditions of optimality for singular control. In this case the order of singularity is $k=1$ and the Legendre-Clebsch condition has the form

$$
(-1)^{k} \frac{\partial}{\partial v} \frac{d^{2 k}}{d t^{2 k}} f_{s} \geq 0,
$$

but:

$$
\frac{\partial}{\partial u} \frac{d^{2}}{d t^{2}} f_{s}=\frac{4}{9} q v^{2} \beta \vartheta e^{2 x / 3}>0,
$$

implying that the necessary condition for optimality of singular control is violated.

This leads to the conclusion that intermediate doses of the drug and radiation are not optimal and that the optimal protocol contains only switches between maximal dose and resting intervals.

Yet another possibility, even more reasonable from the oncologic point of view, is to combine chemotherapy and antiangiogenic therapy. The first attempt in analysis and optimization of the model of such combined therapy was presented in Świerniak (2008). In this case however the two-compartmental model seems to be inadequate. A realistic model should take into account drug resistance of cancer population treated by chemo-toxic agents and phase dependence of the drugs (see e.g. Kimmel and Świerniak, 2006). The simplest model which takes into account emergence of drug resistance in tumor chemotherapy and parallel treatment by antiangiogenic agents may be defined in the following form which combines probably the simplest model of drug resistance (Ledzewicz et al., 2004) with one of the previously discussed models of antiangiogenic therapy:

$$
\begin{aligned}
& \dot{S}=-a S+(1-v)(2-q) a S+r c R, \\
& \dot{R}=-c R+(2-r) c R(1-R / K)+(1-v) q S, \\
& \dot{K} / K=\gamma-\left(\lambda N^{2 / 3}+\mu\right)-\eta u-\xi v,
\end{aligned}
$$

where $S$ and $R(S+R=N)$ denote the average number of tumor cells in sensitive and resistant compartments with growth parameters $a$ and $c$ respectively, $q$ and $r$ denote, respectively, the probability of mutation from sensitive to resistant state and the one for the back mutation, and the other parameters have the same meaning as in the previously analysed models. Although the class of models and optimization problems looks very similar to the ones analysed previously, we are led to a third order system of differential equations and therefore synthesis of the optimal control law is much more difficult. Therefore analysis of this problem will be the subject of our further studies. It is difficult to predict if in this case singular arcs will be present and feasible or only bang-bang controls are optimal. 
Acknowledgements. The author was partly supported by internal SUT grants BK 234/Rau1/2007 and BK 209/Rau1/2008.

The paper is a substantially extended version of the talk presented at the 13th National Conference on Applications of Mathematics in Biology and Medicine, Serpelice, 2007 and published in the Proceedings of that Conference. It is my pleasant duty to thank an anonymous reviewer for his/her valuable remarks which enabled me to improve the paper.

\section{References}

K. Bartha and H. Rieger (2006), Vascular network remodeling via vessel cooption, regression and growth in tumors, J. Theor. Biol. 241, 903-918.

J. Bischoff (1995), Approaches to studying cell adhesion and angiogenesis, Trends Cell Biol. 5, 69-73.

R. J. D'Amato, M. S. Loughnan, E. Flynn and J. Folkman (1994), Thalidomide is an inhibitor of angio-genesis, Proc. Nat. Acad. Sci. USA 91, 4082-4085.

S. Davis and G. D. Yancopoulos (1999), The angio-poietins: Yin and Yang in angiogenesis, Curr. Top. Microbiol. Immunol. 237, 173-185.

J. Denekamp (1993), Angiogenesis, neovascular proliferation and vascular pathophysiology as targets for cancer therapy, Brit. J. Radiol. 66, 181-196

A. d'Onofrio and A. Gandolfi (2004), Tumour eradication by antiangiogenic therapy: analysis and extensions of the model by Hahnfeldt et al. (1999), Math. Biosci. 191, 159-184.

A. Ergun, K. Camphausen, and L. M. Wein (2003), Optimal scheduling of radiotherapy and angio-genic inhibitors, Bull. Math. Biol. 65, 407-424.

J. Folkman (1971), Tumor angiogenesis: therapeutic implications, N. Engl. J. Med. 295, $1182-1186$.

J. Folkman (1972), Antiangiogenesis: new concept for therapy of solid tumors, Ann. Surg. $175,409-416$.

J. Folkman (1995), Angiogenesis inhibitors generated by tumors, Mol. Med. 1, 120-122.

J. Folkman and C. Haudenschild (1980), Angiogenesis in vitro, Nature 288, 551-555.

J. Folkman and M. Klagsburn (1987), Angiogenic factors, Science 235, 442-447.

P. Hahnfeldt, D. Panigrahy, J. Folkman and L. Hlatky (1999), Tumor development under angiogenic signaling: A dynamic theory of tumor growth, treatment response and postvascular dormancy, Cancer Res. 59, 4770-4778.

R. S. Kerbel (1997), A cancer therapy resistant to resistance, Nature 390, 335-340.

R. S. Kerbel (2000), Tumor angiogenesis: past, present and near future, Carcinogenesis 21, 505-515.

M. Kimmel and A. Świerniak (2006), Control theory approach to cancer chemotherapy: benefiting from phase dependence and overcoming drug resistance, in: Tutorials in Mathematical Biosciences III: Cell Cycle, Proliferation, and Cancer, A. Friedman (ed.), Lecture Notes in Math., Math. Biosci. 1872, Springer, 185-222.

M. Klagsburn and S. Soker (1993), VEGF/VPF: the angiogenesis factor found?, Curr. Biol. 3, 699-702.

A. Krener (1977), The high order maximum principle and its application to singular control, SIAM J. Control Optim. 15, 256-293.

J. La Salle and S. Lefschetz (1961), Stability by Liapunov's Direct Method, Academic Press, New York. 
U. Ledzewicz, H. Schättler and A. Świerniak (2004), Finite dimensional models of drug resistance and phase specificity, J. Medical Inf. Techn. 8, IP5-IP13.

U. Ledzewicz and H. Schättler (2005), A synthesis of optimal control for a model of tumour growth under antiangiogenic inhibitors, in: Proc. 44th IEEE CDC and ECC, Seville, 2005, 934-939.

U. Ledzewicz and H. Schättler (2006), Application of optimal control to a system describing tumour anti-angiogenesis, in: Proc. 17 Intern. Symp. MTNS 2006, Kyoto, 478-484.

U. Ledzewicz and H. Schättler (2007), Anti-angiogenic therapy in cancer treatment as an optimal control problem, SIAM J. Control Optim. 46, 1052-1079.

N. V. Mantzaris, S. Webb and H. G. Othmer (2004), Mathematical modeling of tumorinduced angiogenesis, J. Math. Biol. 49, 111-127.

L. S. Pontryagin, V. G. Boltyanskii, R. V. Gamkrelidze and M. Mishchenko (1964), The Mathematical Theory of Optimal Processes, Mac Millan, New York.

A. Świerniak (2007), Control problems arising in combined antiangiogenic therapy and radiotherapy, in: Proc. 5 IASTED Conf. on Biomedical Engineering, Innsbruck, 113117.

A. Świerniak (2008), Modelling of combined anti-angiogenic and chemotherapy, in: Proc. 14th National Conference on Applications of Mathematics in Biology and Medicine, Leszno.

A. Świerniak, G. Gala, A. Gandolfi and A. d'Onofrio (2006a), Optimization of antiangiogenic therapy as an optimal control problem, in: Proc. IASTED Biomech, Acta Press, M. Doblare (ed.), 56-60.

A. Świerniak, A. d'Onofrio and A. Gandolfi (2006), Control problems related to tumor angiogenesis, in: Proc. IEEE IECON 2006, 32 Annual Conf. of the IEEE IES, Paris, $677-681$.

H. D. Thames and J. H. Hendry (1987), Fractionation in Radiotherapy, Taylor \& Francis, London.

N. Weidner (1995), Intramural microvessel density as a prognostic factor in cancer, Amer. J. Path. 147, 9-19.

Institute of Automatic Control

Silesian University of Technology

Akademicka 16

44-100 Gliwice, Poland

E-mail: Andrzej.Swierniak@polsl.pl

Received on 28.5.2008;

revised version on 10.11.2008 